EPJ Web of Conferences 52, 07006 (2013)

DOI: $10.1051 /$ epjconf/20135207006

(C) Owned by the authors, published by EDP Sciences, 2013

\title{
A systematic study of the hybrid experiment at Mt.Chacaltaya
}

\author{
M.Tamada ${ }^{1, a}$, H.Aoki ${ }^{2}$, K.Honda ${ }^{3}$, N.Inoue ${ }^{4}$, N.Kawasumi $^{5}$, N.Martinic ${ }^{6}$, N.Ochi ${ }^{7}$, N.Ohmori ${ }^{8}$, A.Ohsawa ${ }^{9}$, \\ H.Semba ${ }^{10}$, and R.Ticona ${ }^{6}$ \\ ${ }^{1}$ Faculty of Science and Engineering, Kinki University, Higashi-Osaka, Japan \\ ${ }^{2}$ Faculty of Science, Soka University, Hachioji, Tokyo, Japan \\ ${ }^{3}$ Faculty of Engineering, University of Yamanashi, Kofu, Japan \\ ${ }^{4}$ Faculty of Science, Saitama University, Saitama, Japan \\ ${ }^{5}$ Faculty of Education, University of Yamanashi, Kofu, Japan \\ ${ }^{6}$ Insitute de Investigaciones Fisicas, Universidad Mayor de San Andres, La Paz, Bolivia \\ ${ }^{7}$ General Education, Yonago National College of Technology, Yonago, Japan \\ ${ }^{8}$ Faculty of Science, Kochi University, Kochi, Japan \\ ${ }^{9}$ Institute for Cosmic Ray Research, University of Tokyo, Kashiwa, Japan \\ ${ }^{10}$ Faculty of Comprehensive Welfare, Urawa University, Urawa, Japan
}

\begin{abstract}
In the hybrid experiment on Mt.Chacaltaya, we can observe three different components of airshowers, that is, air-shower size, burst-density and high energy families (a bundle of high energy particles). Burst-density in each block of hadron calorimeters are newly recalculated in simulations in oder to compare directly to the experimental data. Energy deposits in the scintillators of the hadron calorimeters are calculated using GEANT4 for every particle, incident upon the hadron calorimeter, in the air-showers simulated using CORSIKA, and are converted into burst-density, taking into consideration the exact structure of experimental hadron calorimeter. We study correlations among three observable components in the air-showers. Correlations between air-shower size and burst-density and those between air-shower size and accompanied family energy can be explained by model calculations by adjusting primary particle composition, the former correlation is in favor of proton-primaries but the latter iron-primaries. No model can describe well observed correlations between burst-density and family energy. That is, the observed family energy accompanied by the air-showers with larger burst-density is systematically smaller than that expected in the simulated events. Effects of a fluctuation in the cross-section of hadronic interactions are studied to settle the disagreement between experimental data and simulations.
\end{abstract}

\section{Introduction}

The "knee" in the cosmic-ray spectrum in the energy region $10^{15} \sim 10^{17} \mathrm{eV}$ is still not well understood. Many of the experimental groups claim that the fraction of heavy primaries increases rapidly beyond the "knee" and the change of the chemical composition of primary cosmicrays is an origin of the "knee"[1-3]. However, the hybrid experiments, operating simultaneously an air-shower array, a hadron calorimeter and an emulsion chamber at Mt. Chacaltaya $(5200 \mathrm{~m}$, Bolivia) [4, 5], have been shown that the characteristics of the observed events in this energy region can not be fully explained simply by changing chemical composition of primary particles. In the hybrid experiments, we can obtain air-shower size, $N_{e}$, from the air-shower array data, particle-density, $n_{b}$, which are closely connected to the hadron component in the airshower, from hadron calorimeter (burst detector) and the energy and geometrical position of individual high energy electromagnetic particles of atmospheric families by the

\footnotetext{
ae-mail: tamada@ele.kindai.ac.jp
}

emulsion chamber. Correlations between air-showers and accompanying families were studied by comparing experimental and simulated data using Chacaltaya data [6, 7] together with the data of the other two hybrid experiments, Tibet ASy (Yang-bajing , 4300m, China)[1, 2] and TienShan (3340m, Kazakhstan)[8]. Although details of the experimental procedure are different in these hybrid experiments the results are found to be almost the same[7]. In the papers $[9,10]$ we have also shown some results on the correlation between burst-density and accompanied family energy observed by the Chacaltaya hybrid experiment. It is found that the experimental data are not well explained by the current model calculations. In the present paper, we use more accurate calculations based on the GEANT4 simulation[11] for the burst-density and compare the Chacaltaya data with improved simulated data.

\section{Hybrid experiment at Mt.Chacaltaya}

The air-shower array covers a circular area within a radius about $50 \mathrm{~m}$ by 35 plastic scintillation detectors to mea- 
sure the lateral distribution of electron density of the airshowers. In the center of the air-shower array, 32 blocks of emulsion chambers $\left(0.25 \mathrm{~m}^{2}\right.$ each $)$ are installed. Each block of the emulsion chamber consists of 30 lead plates each of $0.5 \mathrm{~cm}$ thick and 14 sensitive layers of X-ray film which are inserted after every $1 \mathrm{~cm}$ lead. The total area of the emulsion chambers is $8 \mathrm{~m}^{2}$. Hadron calorimeters with plastic scintillator of $5 \mathrm{~cm}$ thick are installed underneath the respective blocks of the emulsion chamber. A $2 \mathrm{~cm}$ thick iron support is inserted between the emulsion chamber and the hadron calorimeter. Details of the Chacaltaya hybrid experiment are described in Refs.[4, 5]

\section{Simulations}

\subsection{Air-showers}

For generating extensive air-showers and families we use the CORSIKA simulation code (version 6.980) [12] employing the QGSJET model (QGSJET01c)[13] and the EPOS model (EPOS 1.99) [14] for the cosmic-ray nuclear interaction. Primary particles of $E_{0} \geq 10^{15} \mathrm{eV}$ are sampled respectively from the power low energy spectrum of integral power index -1.8 , for pure protons and pure irons, and also from the energy spectrum of primary cosmic rays with proton dominant and heavy dominant chemical composition ${ }^{1}$. The thinning energy in the calculations in the air-showers is fixed to be $10 \mathrm{GeV}$. Shower size, $N_{e}$, at the observation level is calculated by using NKG option in the simulation. Here we use default values in the CORSIKA code for the low energy cut-off of the particles, i.e., $E_{\text {cut }}=0.3 \mathrm{GeV}$ for hadrons and muons and $E_{\text {cut }}=0.003$ $\mathrm{GeV}$ for electromagnetic particles. Air-shower center is randomly sampled within a area of $\pm 2.5 \mathrm{~m}$ in $\mathrm{X}$ and $\mathrm{Y}$ direction from the center of 32 hadron calorimeters.

\subsection{High energy showers in the atmospheric families}

For high energy (e,y)-particles and hadrons of $E \geq 1$ $\mathrm{TeV}$ in the atmospheric families arriving at each emulsion chamber, we calculate further nuclear and electromagnetic cascade development inside the chamber taking into account the exact structure of the emulsion chamber. We use the QGSJET model for hadron-Pb interactions and a code formulated by Okamoto and Shibata for electromagnetic cascade[15]. The electron number density under every 1 $\mathrm{cmPb}$ is converted into spot darkness of the X-ray film. Then the energy of each shower is re-estimated from the shower transition on spot darkness by applying the procedure used in the experiments.

\footnotetext{
${ }^{1}$ Five species of nucleus (proton, $\mathrm{He}, \mathrm{N}, \mathrm{Mg}, \mathrm{Fe}$ ) are assumed in both chemical compositions. The fraction of those nucleus are assumed to be $0.42,0.16,0.16,0.14,0.12$ respectively in proton dominant model and $0.17,0.10,0.18,0.15,0.40$ in heavy-dominant model at $E_{0}=10^{15}-10^{16}$ $\mathrm{eV}$ and the fraction of heavy primaries increases a little at higher energies in both chemical compositions.
}

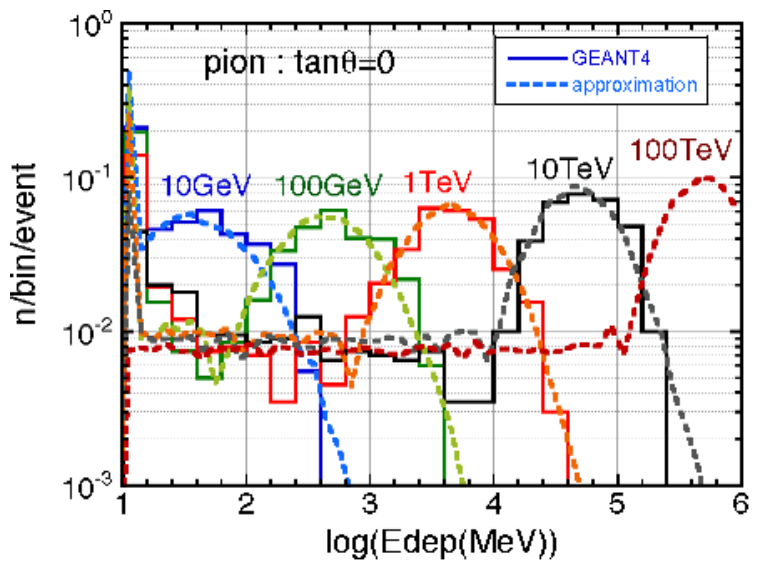

Figure 1. Distribution of energy deposit, $E_{d e p}$, in the plastic $5 \mathrm{~cm}$ thick scintillator of the hadron calorimeter for pions with vertical incidence. Histograms are results obtained by the GEANT4 simulation code and dashed-curves are those obtained by sampling from approximated numerical functions.

\subsection{Calculation of the burst-density}

Hadron calorimeters detect a bundle of charged particles, which are produced in the emulsion chamber material mainly by the hadron component in the air-shower through the local nuclear interactions. We use GEANT4 code[11] with QGSP model for hadronic interactions for calculating the burst-density. We calculate nuclear and electromagnetic cascades in the emulsion chamber and the energy deposit in the $5 \mathrm{~cm}$ thick plastic scintillator is estimated for all the charged particles (mainly electrons and positrons) and $y$-rays in the cascade ${ }^{2}$. Actually, the energy deposit is calculated for the hadrons (pions, protons, neutrons and kaons) and also muons and $e, \gamma$ with 5 different energies of $10 \mathrm{GeV}, 100 \mathrm{GeV}, 1 \mathrm{TeV}, 10 \mathrm{TeV}$ and $100 \mathrm{TeV}$, and 5 different zenith tangent of arrival direction, $\tan \theta=0.0,0.2,0.4,0.6,0.8$. In Figure 1 we show examples of distributions of the energy deposit in the scintillator obtained by using GEANT4 code in case of pions with vertical incidence. The distribution of the energy deposit obtained by the GEANT4 simulations are approximated by numerical functions. In the figure, also shown are the distributions of the energy deposit obtained by sampling from these numerical functions which approximate GEANT4 results. The distributions agree well with those obtained by full simulations using GEANT4. In order to save computing time, the energy deposit is sampled from the numerical functions for every particle incident upon the emulsion chamber and is converted into a particle number using average energy loss $(=10 \mathrm{MeV})$ of a single muon in the $5 \mathrm{~cm}$ thick plastic scintillator. Finally we get the burst-density, $n_{b}$, the number of particles per $0.25 \mathrm{~m}^{2}$, in each block of 32 hadron calorimeters. Details of the procedure for the calculations are given in the paper[16].

\footnotetext{
${ }^{2}$ Gamma-rays also gives some energy deposit in the scintillator by electromagnetic interactions. The scintillator response of gamma-rays are also taken into accounts.
} 


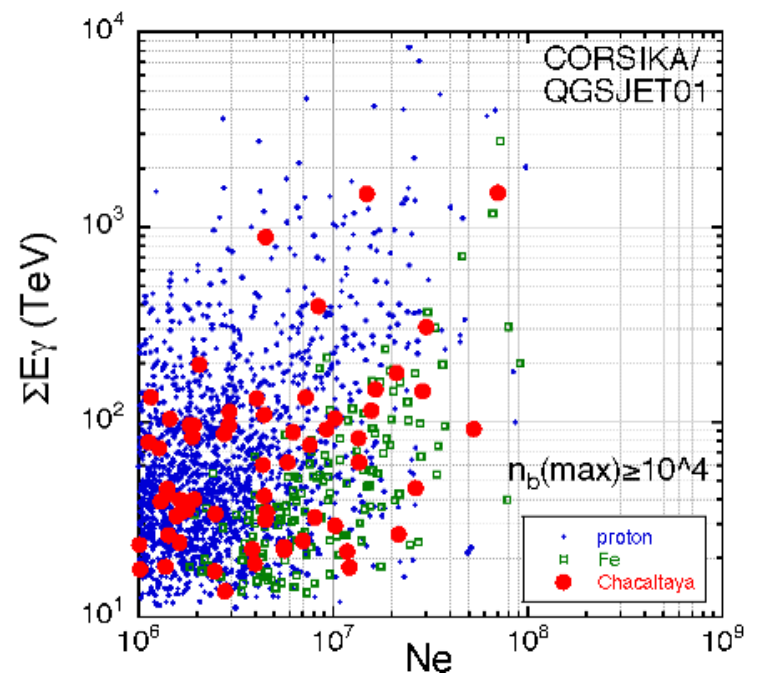

Figure 2. Correlation diagram on shower size, $\mathrm{Ne}$, and associated family energy, $\Sigma E_{\gamma}$ for the events with maximum burstdensity $n_{b}^{\max } \geq 10^{4}$. Large solid circles are for the Chacaltaya data. Small dots and open squares are for proton primaries and for iron primaries, respectively, of the simulated events using CORSIKA with QGSJET01 model.

\section{Selection of the events}

We define $n_{b}^{\max }$ as the largest burst-density among 32 blocks of hadron calorimeters. In the following we pick up the events which satisfy the following criteria;

- $N_{e} \geq 10^{6}$,

- $n_{b}^{\max } \geq 10^{4}$,

- $R_{A S-B S} \leq 1 \mathrm{~m}$,

where $R_{A S-B s}$ is the distance between the burst center and the air-shower center,

- more than 10 among 32 blocks have burst-density $n_{b} \geq$ 100.

The burst center is determined by the algorithm described in Ref.[5]. In the Chacaltaya data, 1,037 events satisfy the above criteria in $\sim 40 \mathrm{~m}^{2}$ year exposure of hadron calorimeters. Among them 62 events are accompanied by high energy atmospheric families of $\Sigma E_{\gamma} \geq 10 \mathrm{TeV}\left(n_{\gamma} \geq 5\right.$ with $E_{\min }=2 \mathrm{TeV}$ ).

\section{Characteristics of air-showers and families}

Figure 2 shows a correlation diagram on the air-shower size, $\mathrm{Ne}$, and the energy sum, $\Sigma E_{\gamma}$ of the associated atmospheric families. The average family energy of the events from iron-primaries is systematically smaller than that from proton-primaries. The experimental data are well described by a mixture of proton- and iron-primaries, and become close to those expected by iron-primaries with increasing shower-size, $\mathrm{Ne}$. The results agree well to the data of Tibet ASy[17] and of Tien-Shan experiment[7].

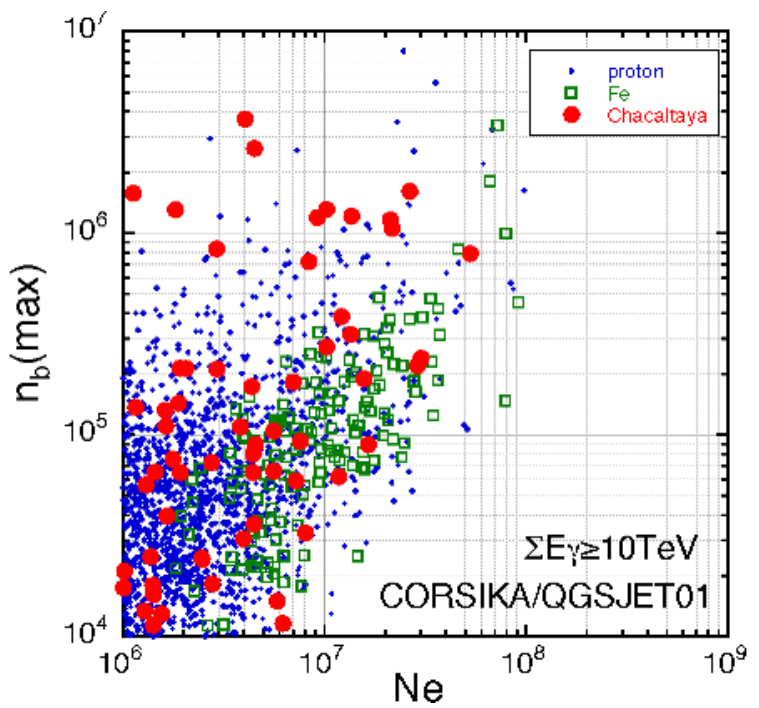

Figure 3. Correlation diagram on shower size, $\mathrm{Ne}$, and maximum burst density, $n_{b}^{\max }$ for the events associated by an atmospheric families of $\Sigma E_{\gamma} \geq 10 \mathrm{TeV}$. Symbols are same as those in Figure 2.

\section{Characteristics of air-showers and bursts}

Figure 3 shows a correlation diagram on air-shower size, $N_{e}$, and the maximum burst-density among 32 blocks of the event, $n_{b}^{\max }$, for the events which accompany an atmospheric family of $\Sigma E_{\gamma} \geq 10 \mathrm{TeV}$. The experimental data are well described by proton-primaries even in the larger shower-size region, contrary to the argument of the previous section ${ }^{3}$.

\section{Characteristics of bursts and families}

Figure 4 shows a correlation diagram between $n_{b}^{\max }$ and accompanying family energy $\Sigma E_{\gamma}$. The experimental data are compared with those of simulated data of protonand of iron-primaries by CORSIKA using QGSJET01 and EPOS1.99 models. As naturally expected, the family energy is roughly proportional to $n_{b}^{\max }$ in the simulated data irrespective of the primary particles though the family energy of the events coming from iron-primaries are smaller than that from proton-primaries. We can see a lot of the experimental data are located far from the simulated events in the diagram. The family energy in the experimental data is systematically smaller than that of simulated data in the events with larger burst-density, $n_{b}^{\max }>\sim 10^{6}$.

The integral spectra of family energy observed by the present hybrid experiment is smoothly connected to that by the emulsion chamber experiment of Brazil-Japan collaboration[18]. Then we can conclude the smaller family energy in the experimental data is not due to the sys-

\footnotetext{
${ }^{3}$ The Tibet AS $\gamma$ group analyzed correlations between air-shower size, $N_{e}$ and burst size, $\Sigma n_{b}$ for all the events including those unaccompanied by a family. They conclude that the experimental distribution on $2 n_{b} / N_{e}$ is well described by assuming heavy-dominant chemical composition of primary particles.
} 

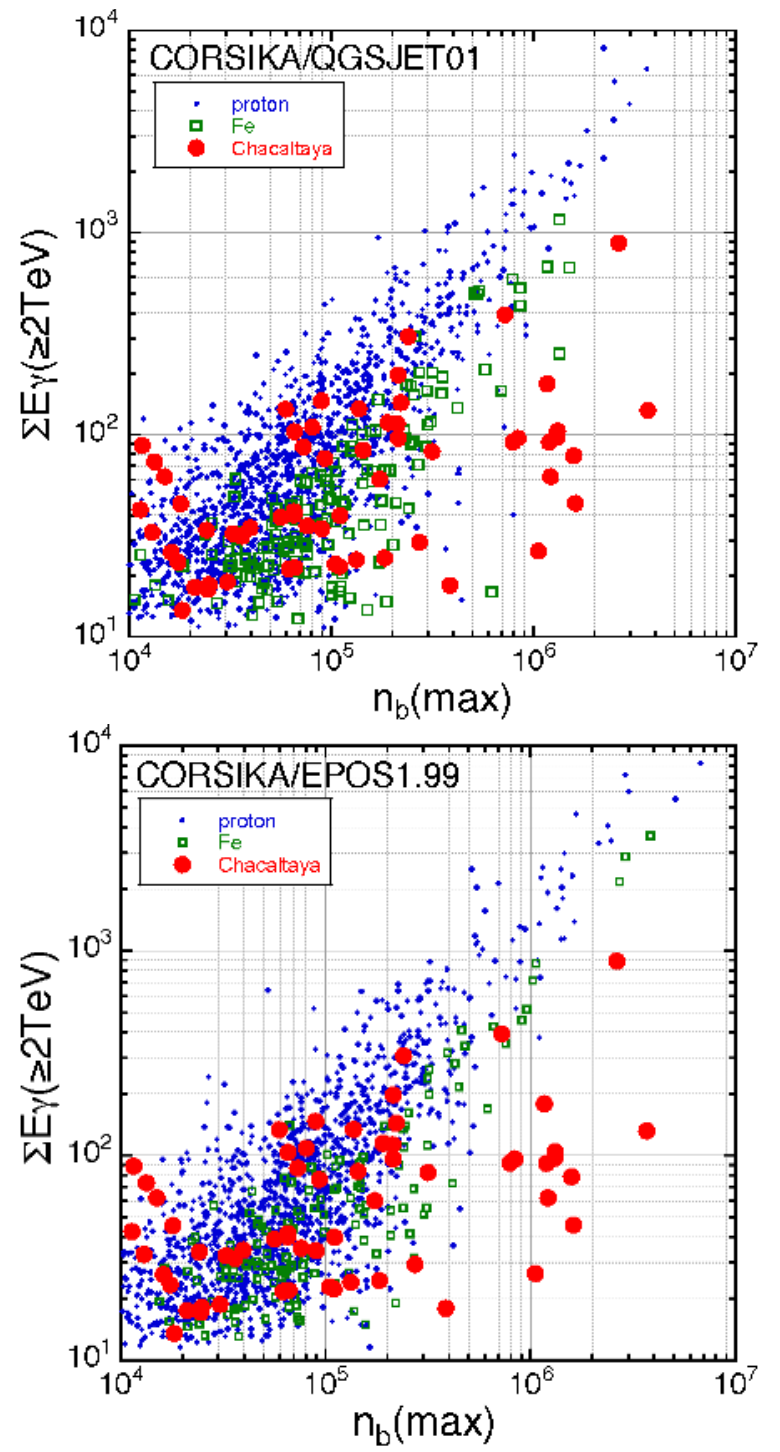

Figure 4. Correlation diagram between $n_{b}^{\max }$ and family energy $\Sigma E_{\gamma}$ in the burst-triggered-families in the air-showers of $10^{6} \leq N_{e}<10^{8}$. Comparison with simulated results of CORSIKA/QGSJET01 (upper) and of CORSIKA/EPOS1.99 (lower). Symbols are same as in Figure 2.

tematic underestimation of the shower energy in the emulsion chamber. An overestimation of the burst-density, especially beyond the region of $n_{b}^{\max } \geq 10^{5}$, is also ruled out, because the distribution on $n_{b}^{\text {max }} / N_{e}$ is well described by the simulations.

No analysis on bursts and families is not yet provided by Tibet ASy and Tien-Shan experiments.

\section{Effect of fluctuations in hadron-Air interaction}

It looks as if the fluctuation of event by event is much more in the experimental data than in simulations as seen in Figure 4. One of the possible origin of the observed large fluctuation could be due to a fluctuation in crosssection of hadronic interaction. In the paper of Wilk

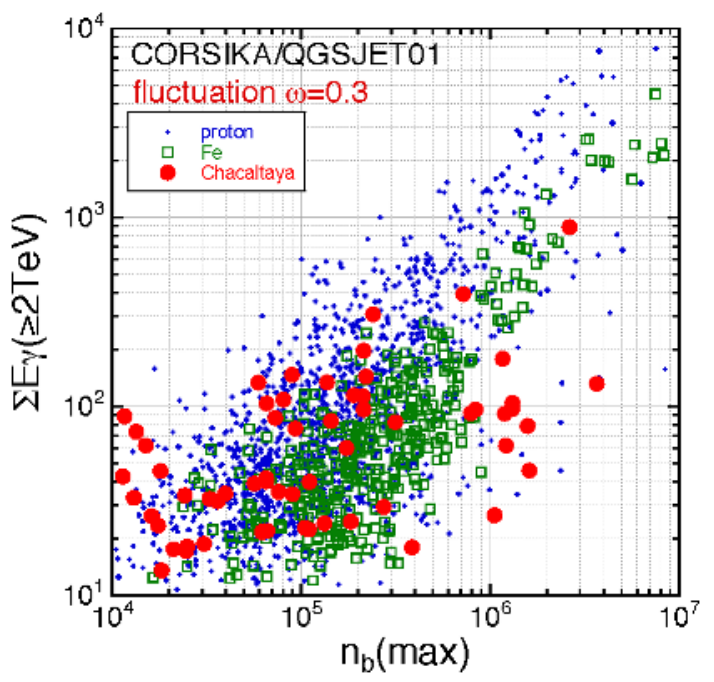

Figure 5. Correlation diagram between $n_{b}^{\max }$ and family energy $\Sigma E_{\gamma}$ in the burst-triggered-families in the air-showers of $10^{6} \leq N_{e}<10^{8}$ in case of introducing fluctuation in the hadronAir interaction in the simulations, with a parameter of relative fluctuation $\omega=0.3$.

and Wlodarczyk[19], non-exponential decrease of showerstarting points of the hadronic showers observed by Pamir thick lead emulsion chamber, one of the unusual phenomena in cosmic-ray interactions, was explained by introducing intrinsic fluctuations in cross-section of hadronic interaction[20]. Here we introduce the same fluctuation of the cross-section in high-energy hadron-air interactions and study the effect to the correlation between bursts and families observed by the hybrid experiment. We apply the same procedure used in their paper [19], that is, the crosssection, $\sigma(E)$, of hadron-air is sampled from the uniform distribution in the interval $\left[1-\sqrt{3 \omega} \sigma_{0}(E), 1+\sqrt{3 \omega} \sigma_{0}(E)\right]$. Here $\sigma_{0}(E)$ is the mean cross-section at the energy $E$ and $\omega$ is the relative fluctuation of the cross-section. We apply these modification in the CORSIKA simulation code and calculate air-showers, bursts and high energy atmospheric families. The results are shown in Figure 5 for the case of $\omega=0.3$. The simulated data scattered out more widely when the fluctuation of the cross-section is included in the calculations, though the frequency to observe the events with larger $n_{b}^{\max }$ and smaller $\Sigma E_{\gamma}$ at the same time is still smaller in simulations than that in the experiment.

Figure 6 shows energy fraction of hadron component and those of electromagnetic component at the observation level in the air-showers induced by primary protons of energy $E_{0}$ for two different threshold energy of particles. The fraction of hadron component, $\Sigma E_{h} / E_{0}$, becomes larger when the fluctuation of the hadron-Air cross-section exists, around two (four) times larger for $E_{h} \geq 10 \mathrm{GeV}$ $(1 \mathrm{TeV})$. In contrast to the hadron component, the energy fraction of the electromagnetic component, $\Sigma E_{e, \gamma} / E_{0}$, is almost unchanged even when cross-section fluctuation is taken into accounts. This is a reason why inclusion of the 

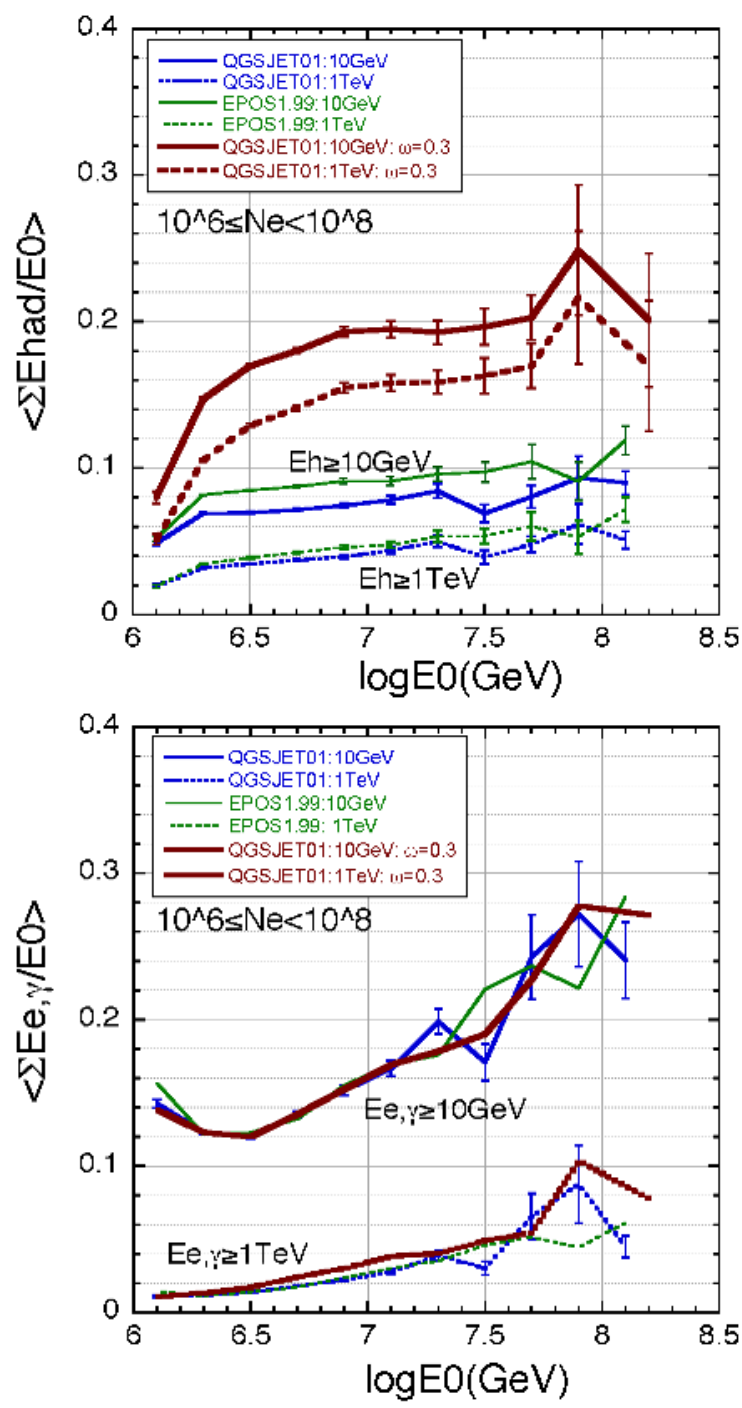

Figure 6. Energy fraction of hadron component (upper) and e, $\gamma$ component (lower) in the air shower induced by a primary proton of energy $E_{0}$. Solid lines are for $E_{h_{s} e_{y},} \geq 10 \mathrm{GeV}$ and dashed lines are for $E_{h_{e}, e_{y}} \geq 1 \mathrm{TeV}$. Thick lines are for QGSJET01 model and thin lines are for EPOS1.99 model. Bold lines are for the case including fluctuation in the hadron-Air interactions in QGSJET01 model.

fluctuation of cross-section make more events with larger burst density.

\section{Summary}

The correlation between air-shower size and associated family energy shows that the average family energy of the experimental data is considerably smaller than that of simulations of proton- primaries in the shower size region of $N e \geq 10^{7}$ and the experimental data are close to those expected in case of Fe-primaries (see Figure 2). On the contrary, the correlation between air-shower size and associated burst-density shows that a considerable number of air-showers induced by proton-primaries accompany large burst-density which are not seen in the iron-induced airshowers, and the experimental data are close to those ex- pected in case of proton-primaries (see Figure 3 ). The contradiction of the above two arguments is well seen in the correlation digram between burst-density and family energy and no model can describe the observed correlation (see Figure 4).

Some changes of the characteristics of particle production may cause the discrepancy between experimental and simulational data, but recent results of the LHCf experiment show the production spectra of secondary particles in p-p collisions at $\sqrt{s}=7 \mathrm{TeV}$ are not much different from those assumed in current simulation models though no model can describe well the LHCf data[21]. As an another possibility, we considered the effect of the fluctuation in the cross-section of hadron-air interactions. It is found that the disagreement between experimental and simulational data becomes smaller when the cross-section fluctuation is taken into accounts.

\section{References}

[1] Tibet AS $\gamma$ Collaboration (M.Amenomori et al.), Phys. Rev. D 62 (2000) 112002-1, 072007-3

[2] Tibet AS $\gamma$ Collaboration (M.Amenomori et al.), Phys. Lett. B 632 (2006) 58

[3] J.Blumer, R.Engel and J R.Horandel, Prog. in Part. Nucl. Phys. 63 (2009) 58

[4] N.Kawasumi et al., Phys. Rev. D 53 (1996) 3534

[5] C.Aguirre et al., Phys. Rev. D 62 (2000) 032003

[6] H.Aoki et al., Proceedings of 30th ICRC, Merida (2007), Vol.4, p.23

[7] S.P.Besshapov et al., Nucl. Phys. B (Proc. Suppl.) 196(2009) 118; Proceedings of 31th ICRC, Lodz (2009) \#0214

[8] S.B.Shaulov, AIP Conf. Proc. 276 (1992) 94

[9] M.Tamada, Proc. 16th Int. Symp. on Very High Energy Cosmic Ray Interactions, Fermilab eConf C1006284 (http://www.slac.stanford.edu/econf/C1006284) C27

[10] H.Aoki et.al., Proceedings of 32th ICRC, Beijing (2011) Vol.1,p205

[11] S.Agostinlli et al. Geant4 collaboration, Nucl. Inst. and Meth. A 506(2003) 250

[12] D.Heck, J.Knapp, J.N.Capdevielle, G.Schatz and T.Thouw, Fortshungzentrum Karlsruhe, FZKA 6019 (1998)

[13] N.N.Kalmykov and S.S.Ostapchenko, Yad. Fiz. 56 (1993) 105

[14] K.Werner, F.M.Liu and T.Pierog, Phys. Rev. C 74 (2006) 044902

[15] M.Okamoto and T.Shibata, Nucl. Instr. and Meth. A 257 (1987) 155

[16] T.Yamasaki and M.Tamada, Proceedings of 32th ICRC, Beijing (2011) Vol.1,p216

[17] M.Shibata, http://www.icrr.u-tokyo.ac.jp/emtibet-exp /2006-08-ICRR/05-Shibata.pdf

[18] C.M.G.Lattes, Y.Fujimoto and S.Hasegawa, Phys. Rep. Vol. 65 (1980) 151

[19] G.Wilk and Z.Wlodarczyk, Phys. Rev. D50 (1994) 2318

[20] B.Blättel et al., Phys. Rev. D47 (1993) 2761

[21] O.Adriani et al. ( LHCf Collaboration), Phys. Lett. B703 (2011) 128 